\title{
Producing Massive Neutral Intermediate Vector Bosons with Existing Accelerators (*)
}

\author{
C. Rubbia and P. McIntyre \\ Department of Physics \\ Harvard University \\ Cambridge, Massachusetts 02138 \\ and \\ D. Cline \\ Department of Physics \\ University of Wisconsin \\ Madison, Wisconsin 53706
}

\begin{abstract}
We outline a scheme of searching for the massive weak boson $\left(M=50-200 \mathrm{GeV} / \mathrm{c}^{2}\right)$. An antiproton source is added either to the Fermilab or the CERN SPS machines to transform a conventional $400 \mathrm{GeV}$ accelerator into a $p \bar{p}$ colliding beam facility with $800 \mathrm{GeV}$ in the center of mass $\left(E_{\text {eq }}=320,000 \mathrm{GeV}\right)$. Reliable estimates of production cross sections along with a high luminosity make the scheme feasible.
\end{abstract}

Submitted to Physical Review Letters March 1976 
The past ten years have seen remarkable progress in the understanding of weak interactions. First there is the experimental discovery of $\Delta S=0$ weak neutral currents, ${ }^{l}$ which when contrasted with the previous limits on $\Delta S=1$ neutral current decay processes ${ }^{2}$ leads to the suggestion of additional hadronic quantum numbers in nature. 3 strong evidence now exists for new hadronic quantum numbers that are manifested either directly ${ }^{4,5}$ or indirectly. 6 The experimental discoveries are complemented by the theoretical progress of unified gauge theories. 7,8 These developments lead to the expectation that very massive intermediate vector bosons $\left(50-100 \mathrm{GeV} / \mathrm{c}^{2}\right)$ may exist in nature. 7,8 The search for these massive bosons require three separate elements to be successful: a reliable physical mechanism for production, very high center of mass energies, and an unambiguous experimental signature to observe them. In this note we outline a scheme which satisfies these requirements and that could be carried out with a relatively modest program at existing proton accelerators.

We first turn to the production process. We concentrate on neutral bosons because of the extremely simple experimental signature and because production is largely dominated by a single production resonant pole in the particle-antiparticle cross section. The best production reaction would of course be:

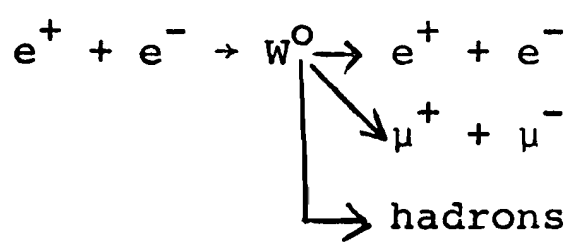

where a sharp resonance peak is expected for $2 E_{e^{+}}=2 E_{e^{-}}=M$. In the 
Breit-wigner approximation near its maximum we get:

$$
\sigma\left(e^{+} e^{-} \rightarrow w^{0}\right) \simeq \frac{3}{4} \pi \hbar^{2} \frac{\Gamma_{i} \Gamma}{(2 E-M)^{2}+\frac{\Gamma^{2}}{4}}
$$

where $\Gamma_{i}, \Gamma$ are the partial width to the initial $e^{+} e^{-}$state and the total width, respectively. The decay widths into $e^{+} e^{-}\left(\right.$and $\left.\mu^{+} \mu^{-}\right)$ pairs can be calculated in the first order of the semi-weak coupling constant: $\Gamma_{\mathrm{e}^{+} \mathrm{e}^{ \pm}} \cong \Gamma_{\mu^{+} \mu^{-}}=1.5 \times 10^{-7} \mathrm{M}_{\mathrm{W}}^{3}(\mathrm{GeV})$. For $\mathrm{M}=100 \mathrm{GeV}$, $\Gamma_{e^{+} e^{-}} \simeq 150 \mathrm{MeV}$, which is surprisingly large. The total width is related to the above quantity by the branching ratio $\mathrm{B}_{e^{+}} e^{-}=\Gamma_{e^{+}} e^{-/ \Gamma}$ which is unknown. Crude guesses based on quark models suggest $B_{e^{+} e^{-}} \simeq 1 / 10$, giving $\Gamma=1.5 \mathrm{GeV}$ or $\Gamma / 2 \mathrm{E}=1.58$ for $M=100 \mathrm{GeV} / \mathrm{c}^{2}$. At the peak of the resonance, $\sigma\left(e^{+} e^{-} \rightarrow W^{\circ}, 2 E=M\right)=3 \pi z^{2} B_{i} \simeq$ $2.10^{-31} \mathrm{~cm}^{2}$. Neutrino experiments ${ }^{9}$ have found that $\mathrm{M}_{\mathrm{W}^{+}}>20 \mathrm{GeV} / \mathrm{c}^{2}$. Therefore, if $M_{W^{0}} \sim M_{W^{+}}$, the neutral intermediate boson is out of reach of existing $e^{+} e^{-}$storage rings.

A more realistic production process is the one initiated by proton-antiproton collisions:

$$
p+\bar{p} \rightarrow w^{0}+(\text { hadrons })
$$

which, according to the quark (parton) picture, proceeds by a reaction analog to (1), except that now incoming $e^{+}$and $e^{-}$are replaced with $q$ and $\bar{q}$. Strong support to the idea that $W^{\prime} s$ are directly coupled to spin $1 / 2$ point-like constituents comes from neutrino experiments 10 and from semi-leptonic hadron decays. 11 Furthermore neutrino experiments provide the necessary structure functions and have set limits ${ }^{9}$ ( $>20 \mathrm{GeV}$ ) on any nonlocality in the parton form factor. The main 
difference with respect to $\mathrm{e}^{+} \mathrm{e}^{-}$is that now the kinematics is largely smeared out by the internal motion of $\mathrm{q}^{\prime} \mathrm{s}$ and $\bar{q}^{\prime} \mathrm{s}$. The average center of mass energy squared of the $q-\bar{q}$ collision is roughly ${ }^{12}$ :

$$
\left\langle\mathrm{s}_{\mathrm{q} \overline{\mathrm{q}}}>\sim \mathrm{s}<\mathrm{x}_{\mathrm{q}}>_{\mathrm{p}}<\mathrm{x}_{\overline{\mathrm{q}}}>_{\bar{p} \mathrm{p}}\right.
$$

where $S$ is the center of mass energy squared of the $\bar{p} p$ system and $\left\langle x_{q}>_{p}\left(<x_{\bar{q}}\right\rangle_{p}\right)$ is the mean fractional momentum of $q^{\prime} s\left(\bar{q}^{\prime} s\right)$ in the proton (antiproton). From the neutrino measurements ${ }^{9}$ and $<\mathrm{x}_{\mathrm{q}}>_{\mathrm{p}}=$ $\left\langle x_{\bar{q}}\right\rangle_{\bar{p}}$ we find $\left\langle S_{q \bar{q}}>\sim 0.04 \mathrm{~s}\right.$. For $M=100 \mathrm{GeV} / \mathrm{c}^{2}$ this suggests $S \geq 2 \times 10^{5} \mathrm{GeV}^{2}$ or $\sqrt{\mathrm{S}} \geq 450 \mathrm{GeV}$. The production cross section can be evaluated by folding the (narrow) resonance (2) over the $q$ and $\bar{q}$ momentum distributions:

$$
\sigma\left(q \bar{q} \rightarrow w^{0}+\mu^{+} \mu^{-}\right)=3 \pi \lambda^{2} \frac{\Gamma q \bar{q}}{\Gamma} \cdot \frac{\Gamma \mu \mu}{\Gamma} \cdot \frac{d N}{d E}(E=M): 2 \Gamma
$$

where $\frac{d N}{d E}$ is the probability (per unit of energy) of finding a $q \bar{q}$ collision with center of mass energy $E$, and the other symbols have the same meaning as in (2). Note that $\frac{\Gamma q \bar{q}}{\Gamma} \simeq O(1)$ is a model-dependent parameter. The resultant cross section is $\sigma\left(\mathrm{p} \overline{\mathrm{p}} \rightarrow \mathrm{W}^{\circ}+\right.$ hadrons $\rightarrow \mu^{+}+$ $\mu^{-}+$hadrons $) \simeq 6 \pi \lambda^{2} \frac{\Gamma \mathrm{q} \overline{\mathrm{q}}}{\Gamma} \frac{\mathrm{dN}}{\mathrm{dE}}(E=\mathrm{M}) \cdot \Gamma_{\mu \mu} \cong 10^{-32} \mathrm{~cm}^{2}$. The numerical value is given for $M=100 \mathrm{GeV} / \mathrm{c}^{2}, \sqrt{\mathrm{S}}=500 \mathrm{GeV}$ and $\frac{\Gamma_{\mathrm{q}} \overline{\mathrm{g}}}{\Gamma}=1 / 2$. This derivation of the cross section exposes the basic simplicity of the assumptions and gives the order of magnitude of the expected cross section. More sophisticated calculations give similar results. ${ }^{12}$ We note that calculations of $\mathrm{W}^{ \pm}$production in proton-proton collisions are very uncertain in contrast to the present one due to the apparent small antiparton content in the nucleon and the unknown distributions of this component. 13

We turn now to the question of the experimental observation. 
The cleanest experimental signature for the program outlined here is:

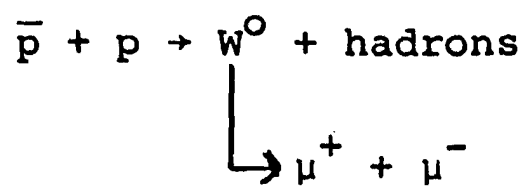

with the observation of a peak in the $\mu^{+} \mu^{-}$invariant mass spectrum with the cross section of equation (3). A modest magnetized iron detector system is adequate to detect the high energy decay muons $\left(P_{\mu} \sim 50 \mathrm{GeV}\right)$ in the center of mass system. Electromagnetic production of $\mu^{+} \mu^{-}$pairs is expected to be suppressed by a factor of $\approx\left(\alpha^{2} / G^{2} M_{W}^{4}\right)$. Note that a similar suppression is expected to hold for any hadronic vector meson. Note also that the production and decay of charged vector bosons is more problematic since the decay sequence

$$
\bar{p}+p+w^{+}+x
$$

leads to one muon and a missing neutrino which is difficult if not impossible to detect. In many previous discussions it has been assumed that the $\mathrm{w}^{+}$would be produced with very little transverse momentum with respect to the incident beam direction and therefore the transverse momentum of the decaying $\mu$ would exhibit a sharp peak at $\mathrm{p}_{\mu \perp} \sim \mathrm{M}_{\mathrm{W}} / 2 .{ }^{14}$ Present evidence in case of the production of massive strongly interacting vector bosons (i.e., $\mathrm{J} / \psi$ ) indicate that the parent is produced at relatively large $p_{\perp}$ and therefore the Jacobian peak is largely smeared out. ${ }^{15}$ There is no obvious reason why the production of massive intermediate vector bosons should not follow the same behavior. ${ }^{16}$ without a sharp structure in the $p_{\mu \perp}$ distribution, a crucial experimental signature for the $\mathrm{w}^{+}$is absent.

We now briefly outline the scheme of transforming an existing 
proton accelerator into high luminosity p $\bar{p}$ colliding beams ${ }^{17}$ using standard vacuum ( $p \simeq 10^{-7}$ Torr) and the separate function magnet system. The main elements are (1) an extracted proton beam to produce an intense source of antiprotons at $3.5 \mathrm{GeV} / \mathrm{c}$, and (2) a small ring of magnets and quadrupoles that guides and accumulates the $\bar{p}$ beam, (3) a suitable mechanism for damping the transverse and longitudinal phase spaces of the $\overline{\mathrm{p}}$ beam (either electron cooling ${ }^{18}$ or stochastic cooling ${ }^{19}$ ), (4) an R.F. system that bunches the protons in the main ring and in the cooling ring, (5) transport of the "cooled" R.F. bunched $\bar{p}$ beam back to the main ring for injection and acceleration. A long straight section of the main ring is used as p $\bar{p}$ interaction region. A schematic drawing of these elements for the FNAL accelerator is presented in Fig. 1. The main parameters of the scheme are summarized in Table $I$.

The luminosity for two bunches colliding head-on is estimated using the relation

$$
I=N_{p} N_{\bar{p}} \phi / a
$$

where $\mathrm{N}_{\mathrm{p}}$ and $\mathrm{N}_{\mathrm{p}}$ are the number of protons and antiprotons circulating in the machine, respectively, $\phi$ is the revolution frequency and a 15 the effective area of interaction of the two beams. $\mathrm{N}_{\mathrm{p}}$ is taken as $10^{12}$ protons in one R.F. bunch. The value of $\mathrm{N}_{\mathrm{p}}$ is limited by the maximum allowed beam-beam tune shift $\left(N_{p}=10^{12}\right.$ for $\left.\Delta v=0.01\right)$. We have verified the longitudinal stability of the bunch, the phase area growth due to R.F. noise, the transverse wall instability, the headtail effect and non-linear resonances, including those arising from beam-beam interactions. None of these effects appears to be important. 20 we note that $\mathrm{N}_{\mathrm{p}}=10^{12}$ corresponds to $\mathrm{i}_{\mathrm{av}}=10 \mathrm{~mA}$ and 
$i_{\text {peak }}=25 \mathrm{~A}$ for $\ell_{\text {bunch }}=2.5 \mathrm{~m}$ and that the Brookhaven AGs currently accelerates twelve bunches of similar characteristics.

The production of antiprotons at $3.5 \mathrm{GeV}$ is done with protons from the same accelerator and with an overall efficiency $\bar{p} / \mathrm{p} \simeq 4 \times 10^{-6}$. In order to reach $\mathrm{N}_{\overline{\mathrm{p}}}=3 \times 10^{10}$ we need 750 pulses with $10^{13} \mathrm{ppp}$. About 10 seconds must elapse between pulses in order to clear away the freshly injected antiprotons. 21 Therefore the formation of $\bar{p}^{\prime} s$ would take of the order of few hours.

In order to make the beam as small as possible one can reduce the value of the betatron function in the collision point $\left(\beta_{v} \simeq \beta_{h}=3.5 m\right)$ and make the momentum compaction factor close to zero. $^{22}$ Then for standard beam emittances ${ }^{23}$ and $E_{p}=E_{\bar{p}}=250 \mathrm{GeV}$ we calculate $\mathrm{L}=5 \times 10^{29} \mathrm{~cm}^{-2} \mathrm{sec}^{-1}$ for $\mathrm{N}_{\overline{\mathrm{p}}}=3 \times 10^{10}$. In order to observe one event/hour at our estimated cross section we require a Iuminosity of $3 \times 10^{28} \mathrm{~cm}^{-2} \mathrm{sec}^{-1}$. If the more pessimistic cross section of $10^{-33} \mathrm{~cm}^{2}$ is used, a luminosity of $3 \times 10^{29} \mathrm{~cm}^{-2} \mathrm{sec}^{-1}$ is needed which is still appreciably less than the calculated value. Finally, the half-life of the luminosity due to beam-gas scattering is about 24 hours for an average residual pressure of $0.5 \times 10^{-7}$ Torr.

We would like to acknowledge Drs. T. Collins, R. Herb, S. Glashow, E. Picasso, G. Petrucci, N. Ramsey, L. Sulak, L. Thorndahl, and $\mathrm{s}$. Weinberg for helpful discussions and suggestions. 
1. MAIN RING (Fermilab)

- Beam momentum

- Equivalent laboratory energy for (p $\bar{p})$

- Accelerating and bunching frequency

- Harmonic number

- R.F. peak voltage/turn

- Residual gas pressure

- Beta functions at interaction point

- Momentum compaction at int. point

- Invariant emittances $\left(\mathrm{N}_{\mathrm{p}}=10^{12}\right)$

- longitudinal

- transverse

- Bunch length

- Design Iuminosity

2. ANTIPROTON SOURCE (Stochastic Cooling ${ }^{21}$ )

- Nominal stored $\bar{p}$ momentum

- Circumference of ring

- Momentum acceptance

- Betatron acceptances

- Bandwidth of momentum stochastic cooling

- Maximum stochastic accelerating R.F. voltage

- Bandwidth of betatron stochastic cooling

- Final invariant emittances $\left(N_{-}=3.10^{10}\right)$

- longitudinal

- transverse
$250(400) \mathrm{GeV} / \mathrm{C}$

133 (341) $\mathrm{TeV}$

$53.14 \mathrm{Mc} / \mathrm{s}$

1113

$3.3 \times 10^{6}$ Volt

$<0.5 \times 10^{-7}$ Torr

$3.5 \mathrm{~m}$

$\sim 0 \mathrm{~m}$

$3 \mathrm{eV} \mathrm{s}$

$50 \pi 10^{-6} \mathrm{rad} \mathrm{m}$

$2.3 \mathrm{~m}$

$5 \times 10^{29}\left(8 \times 10^{29}\right) \mathrm{cm}^{-2} \cdot \mathrm{s}^{-1}$ 


\section{Figure Caption}

Fig. 1. General layout of the $p \bar{p}$ colliding scheme. Protons $(100 \mathrm{GeV} / \mathrm{c})$ are periodically extracted in short bursts and produce $3.5 \mathrm{GeV} / \mathrm{c}$ antiprotons which are accumulated and cooled in the small stacking ring. Then $\bar{p}^{\prime} s$ are reinjected in an R.F. bucket of the main ring and accelerated to top energy. They collide head-on against a bunch filled with protons of equal energy and rotating in the opposite direction. 


\section{$\underline{\text { References }}$}

*Work supported in part by the U. S. Energy Research and Development Administration.

1. F. J. Hasert et al, and A. Benvenuti et al., papers submitted to the Sixth International Symposium, Bonn (1973), F. J. Hasert et al., Phys. Letters $46 \mathrm{~B}, 138$ (1973), A. Benvenuti et al., Phys. Rev. Letters 32, 800 (1974).

2. U. Camerini, D. Cline, W. Fry and W. Powell, Phys. Rev. Letters 13, 318 (1964), M. Bott-Bodenhausen et al., Phys. Letters 24B, 194 (1967).

3. S. L. Glashow, J. Iliopoulos and L. Maiani, Phys. Rev. D2, 1285 ,

4. B. Aubert et al., "Experimental Observation of $\mu^{+} \mu^{-}$Pairs Produced by Very High Energy Neutrinos", in Proceedings of the Seventeenth International Conference on High Energy Physics, London, 1974 and in Neutrinos - 1974, AIP Conference Proceedings No. 22, edited by C. Baltay (American Institute of Physics, New York, 1974), p. 201. A. Benvenuti et al., Phys. Rev. Letters 34,419 (1975), ibid. 34, 597 (1975).

5. J. von Krogh et al., "Observation of $\mu^{-} e^{+} K_{S}^{0}$ Events Produced by a Neutrino Beam", submitted to Phys. Rev. Letters; J. Blietschau et al., "Observation of Muon-Neutrino Reactions Producing a Positron and a Strong Particle", submitted to Phys. Letters.

6. J. J. Aubert et al., Phys. Rev. Letters 33, 1404 (1974), J. E. Augustin et al., P iys. Rev. Letters 33, 1406 (1974). 
7. S. Woinborg, Phys. Rav. Lettors 19, 1264 (1967).

8. A. Salam in Elementary Particle physics (edited by $N$. Svortholm, Almquist and Wiksells, Stockholm, 1968), p. 367.

9. A. Benvenuti et al., "Test of Locality of the Weak Interaction in High Energy Neutrino Collisions" to be submitted to Phys. Rev. Letters (March 1976).

10. See for instance A. de Rujula: Quark Tasting with Neutrinos. Proceedings 1976 Coral Gables Conference, Miami, Florida, January 1976.

11. I. M. Chunet, J. M. Gaillard and M. K. Gaillard, Physics Reports $\underline{4 C}(1972), 5$.

M. Roos, Phys. Letters, 36B (1971) 130.

12. Drell-Yan, Phys. Rev. Letters 25, 316 (1970), Pakvasa, Parashar, and Tuan, Phys. Rev. D10, 2124 (1975), S. M. Berman, Bjorken, Kogut, Phys. Rev. D4, 3388 (1971), G. Altarelli et al., Nucl. Phys. B92, 413 (1975), and R. B. Palmer, Paschos, Samios and Wang, BNL preprint 20634 .

13. A recent estimate of the antiparton content of the nucleon has been obtained using antineutrino scattering data below $30 \mathrm{GeV}$ and is reported by $A$. Benvenuti et al., "Further Data on the High-y Anomaly in Inelastic Antineutrino Scattering", submitted to Phys. Rev. Letters (Feb. 1976).

14. Y. Yamaguchi, Nuovo Cim. 43, 193 (1966); L. Lederman and B. Pope, Phys. Rev. Letters 27, 765 (1971).

15.. B. Knapp et al., Phys. Rev. Letters 34, 1044 (1975), Y. M. Antipov, et al., IHEP 75-125, Serpukhov (1976), F. W. Büsser et al., Phys. Letters 56B, 482 (1975), K. W. Anderson et al., submitted to Phys. Rev. Letters (1975), D. C. Hom et al., submitted to Phys. Rev. Letters (1976). 
16. F. Halzen, private communication.

17. There are also various schemes for producing at modest cost a pp colliding beam machine of sufficient energy to produce the intermediate vector bosons. One such scheme is to collide the Fermilab main ring with the projected energy doubler ring (Cline, Richter, and Rubbia, private communication with R. R. Wilson, 1975); another scheme is to collide the Fermilab main ring with a small ring of $25 \mathrm{GeV}$ protons (J. K. Walker et al., proposal submitted to Fermilab, 1976).

18. G. I. Budker, Atomic Energy 22, 346 (1967).

G. I. Budker, Ya. S. Derbenev, N. S. Dikansky, V. I. Kudelainen, I. N. Meshkov, V. V. Parkhomchuk, D. V. Pestrikov, B. N. Sukhina, A. N. Skrinskiy, Experiments on Electron Cooling, paper presented at the National Conference, Washington, March 1975.

19. L. van der Meer, CERN-ISR-PO/72-31 August 1972 (unpublished). P. Braham et al., NIM 125,156 (1975). L. Thorndahl, CERN-ISR-RF/75 (unpublished).

20. See, for a complete discussion, M. Month, in Proceedings IX International Conference on High Energy Accelerators, SLAC, May 1974, page 593.

21. L. Thorndahl, CERN-ISR, RF/75 and C. Rubbia, report in preparation. 22. An electron target for NAL, in Proceedings 1973 NAL Summer Study, Vol. 2, page 21 .

23. T. Collins, private communication. 


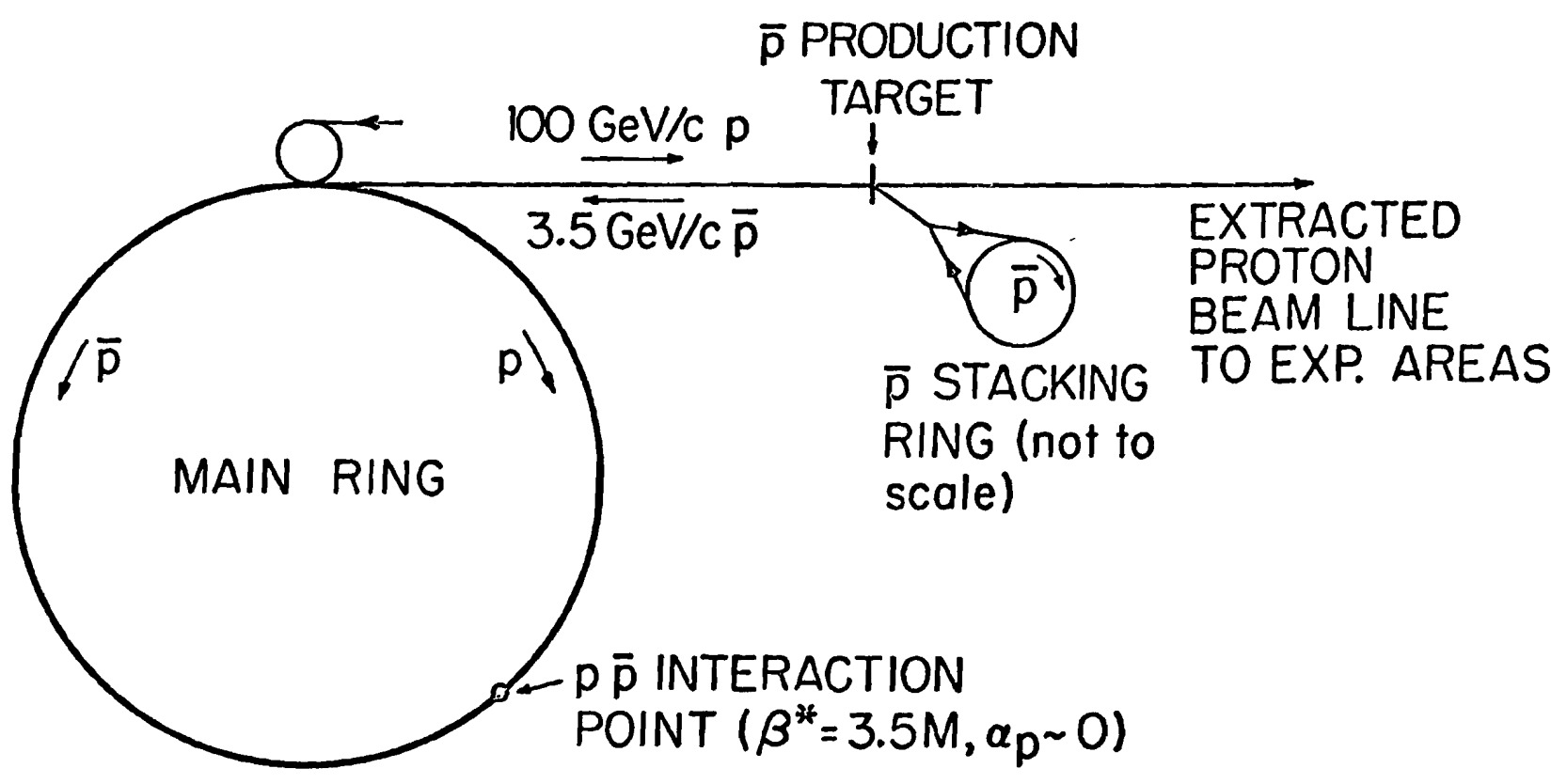

Fig. 1 
